\title{
The Impact of Performance Goals on Cheating Depends on How Performance Is Evaluated
}

\author{
Martin Daumiller \\ University of Augsburg \\ Stefan Janke \\ University of Mannheim
}

\begin{abstract}
We investigated how evaluation standards employed in performance tests affect the impact of performance goals (here focused on appearance) on academic cheating. Thereby, we assumed that appearance goals would lead to increased cheating only if students' performance was presumably evaluated based on results rather than on the strategies they applied to solve questions. A total of 169 university students (68.6\% female) participated in an experimental design with 2 (induced appearance goals vs. no goal induction) $\times 2$ (process-based vs. result-based evaluation standards) between-subject conditions. We assessed cheating using a confederate student observing the participants' behaviors and by measuring whether participants reported that they solved unsolvable questions. Confirming our hypotheses, we found that students were more likely to cheat only when appearance goals were induced and the evaluation standard focused on the results. This new knowledge helps explain the mixed findings regarding how performance goals affect cheating and provides opportunities to reduce cheating in high-stakes testing situations.
\end{abstract}

Keywords: achievement goals, evaluation standards, cheating, academic dishonesty, performance goals

ACADEMIC cheating behaviors, such as passing notes or copying answers during a test, are common at universities. According to survey research, about half to two thirds of university students admit to having engaged in such cheating behaviors at least once during their studies despite knowing that this is largely deemed socially unacceptable (McCabe, 2005; Rettinger, Jordan, \& Peschiera, 2004; Teixeira \& Rocha, 2010). High cheating rates can threaten the validity of test results and, as a consequence, the meaning of grades. The high frequency of academic cheating has prompted researchers to investigate which motivations drive students to engage in these behaviors (see Anderman \& Koenka, 2017, for an overview). Thereby, empirical research supports the notion that academic cheating is detrimental to learning goal striving as it hinders actual knowledge acquisition (e.g., Jordan, 2001; Marsden, Carroll, \& Neill, 2005). It is also highly plausible that appearance goals (i.e., the striving for competence demonstration) may facilitate cheating because cheating can be considered helpful for the attainment of praise for good performance. However, prior findings on this relationship indicate that appearance goals do not always translate into academic dishonesty. Instead, the effect of appearance goals seems to largely depend on further variables (e.g., personal attitudes-Janke, Daumiller, \& Rudert, 2019; social norms-Daumiller \& Janke, 2019). To design test situations that reduce the likelihood of cheating, knowledge about the moderators bound to the test situation is especially necessary. Such knowledge would be particularly important for high-stakes test situations where it may be more suitable to address situated moderators than to reduce appearance goals. To this end, we propose that the extent to which appearance goals lead to increased cheating depends on the evaluation standards employed by the teacher. Specifically, we expect increased cheating by individuals pursuing strong appearance goals when there is a focus placed on the correctness of the answers rather than on the processes that lead to these answers.

\section{The Complex Relationship Between Appearance Goals and Cheating}

As a severe form of academic dishonesty, cheating constitutes a deviation from social norms and may, if detected, have detrimental consequences, such as punishment (Megehee \& Spake, 2008) or social rejection (see Rudert, Ruf, \& Greifeneder, 2019, regarding the association between norm violations and ostracism). Thus, for individuals to cheat, such behaviors must offer rewards that directly connect to their aspired goals. Achievement goal theory (Elliot, 2005; Grant \& Dweck, 2003; Murayama, Elliot, \& Friedman, 2012) provides a suitable framework to explain which personal goals may drive individuals to engage in or refrain from cheating behaviors. By focusing on the end states that individuals prefer to approach or to avoid, this theory seeks 
to describe which goals motivate behavior in achievement situations. Achievement goal researchers have suggested that the striving for competence enhancement (i.e., learning goals sensu Grant \& Dweck, 2003) is particularly relevant for disengagement in academic cheating (Anderman, 2007; Anderman \& Koenka, 2017). The reason for this is that individuals are likely aware that cheating can hinder true learning, which should lead to less cheating if they aspire to expand their competencies. In line with this reasoning, empirical research has shown that learning goals are consistently negatively associated with cheating (e.g., Jordan, 2001; Marsden et al., 2005).

In contrast, research has suggested that (undetected) cheating can be an adaptive strategy to achieve high grades, especially for students who normally struggle to do so (Ramberg \& Modin, 2019). Thus, it could be highly attractive to engage in cheating when striving for appearance goals (i.e., striving for competence demonstration). However, the empirical findings on the association between appearance goals and cheating behavior are inconclusive. A current review of prior research indicated that there were 16 nil findings, 4 (partially) positive associations, and 6 (partially) negative associations (see Daumiller \& Janke, 2019, for a comprehensive summary; see also Krou, Hoff, Hewett, \& Fong, 2019). This mixed pattern of findings suggests that additional variables are relevant for whether appearance goals enhance the likelihood of cheating.

Janke et al. (2019) reported in a sample of university researchers that the strength of the association with selfreported engagement in academic dishonesty (in the form of questionable research practices) is indeed moderated by other intrapersonal variables (here personal attitudes). Although this research was only correlational (and not conducted on the population of university students), the first experimental research by Daumiller and Janke (2019) supported the notion that the effects of appearance goals on cheating depend on additional variables bound to the achievement situation (here observed cheating).

The aforementioned findings from Daumiller and Janke (2019) entail two important insights. First, the research shows that achievement goals are malleable and can be manipulated through experimental procedures. This parallels prior research that has shown that achievement goals are influenced by both internal motivational dispositions (e.g., stable life aspirations; Janke \& Dickhäuser, 2019) as well as external factors (e.g., classroom goal structures evoked by teachers; Lüftenegger, Van De Schoot, Schober, Finsterwald, \& Spiel, 2014). It therefore stands to reason that achievement goal pursuit can be affected through educational means, as also suggested by other successful experimental goal manipulations (e.g., Darnon, Butera, \& Harackiewicz, 2007; Dickhäuser, Buch, \& Dickhäuser, 2011). Second, research shows that students likely rely on social cues (e.g., observing other students cheating) when elaborating about the appropriateness of cheating, which in turn matters for the effects of appearance goals. We acknowledge that it is often difficult for teaching personnel to influence peer norms, which in our opinion justifies a broader search for potentially malleable social cues that might diminish the association between appearance goals and academic cheating. We believe that it is particularly important to focus on aspects of the test itself as teaching personnel have higher control over the content of the test than over social norms during the test. Regarding this, one aspect of the test that can easily be changed is how faculty members assess the performance of their students (i.e., evaluation standards).

\section{Appearance Goals, Cheating, and Performance Evaluation}

Based on the expectancy-value theory of achievement motivation (Wigfield \& Eccles, 2000), we theorize that decisions to engage in (cheating) behaviors depend on a combination of the value ascribed to the result of that action and the expectancy of success. Appearance goals strengthen the degree to which students aim for attaining praise for their competencies (value), which is often articulated through positive competence evaluation (e.g., in the form of grades) by their instructors (Brookhart et al., 2016). Thus, performing well in a given task should have high value for students with strong appearance goals. Usually, expectancy-value approaches consider self-efficacy or related constructs as major influences for students' expectancy to succeed (in cheating; Finn \& Frone, 2004). In the present study, we argue that expectancy to succeed in cheating may also be affected through contextual features that we expect to affect students' expectancy beliefs and, consequently, their cheating behaviors (in line with Dietrich, Viljaranta, Moeller, \& Kracke, 2017).

Instructors control certain contextual features of the achievement situation, such as the evaluation standards that define the rules guiding the attainment of appearance goals. A critical distinction concerning how student performance is evaluated is whether solely the correctness of the answers is considered to be relevant (i.e., focus on results) or the processes and strategies that lead students to the answers (i.e., focus on process). We consider the distinction between these two evaluation standards to be an important factor that could directly influence the extent to which students expect that they can reach their desired outcomes by means of cheating.

We assume that under the condition that instructors strongly value solely the final answers (e.g., correct answers in multiple-choice tests) in performance evaluations, students should consider cheating as, in principle, relatively easy to manage as it only requires simple techniques such as copying the correct results from the answer sheet of another student to acquire a good performance evaluation. In contrast, cheating likely becomes much more complex and its success less certain when instructors focus to a greater degree on the processes that lead to the results (e.g., by awarding points for the 
correct strategy or chain of arguments). Under such circumstances, students would need to copy large pieces of their neighbor's work, and small mistakes in doing so are more likely to impair the whole result.

Furthermore, it is also more likely that students' cheating would be detected if they have to copy or communicate large pieces of information. In this case, success expectations are more uncertain. We therefore assume that students who strongly care about their competence evaluation would be less likely to engage in cheating under such conditions. At this point, it is important to note that expectancy-value theory postulates that expectancies and values are tied together in a multiplicative rather than additive way when predicting engagement in behavior (Nagengast et al., 2011; Trautwein et al., 2012). This implies that even when the value of a certain outcome is high (e.g., the importance of high-performance evaluation because of strong appearance goals), individuals will still not choose to engage in behaviors such as cheating if they are uncertain about whether or not they will succeed. Conversely, if the value ascribed to cheating is low (e.g., when appearance goals are weak), individuals will also not engage in cheating behaviors even if they are certain of their potential success in doing so.

Taken together, we assume that appearance goals will especially lead to cheating when students' performance is evaluated by the end result rather than by the processes that lead to the answers. As both achievement goals and test features can be influenced (by faculty and likely also through experimental manipulations), experimental designs can be considered a promising method to examine their interplay further.

\section{Research Questions}

In the present study, we aimed to investigate the impact of two types of evaluation standards (process vs. result oriented) on the effect of appearance goals on cheating. We expected that appearance goals would induce cheating behavior only when students believed that their performance would be assessed solely by evaluating their end results. We aimed to show this moderation effect in a naturalistic experiment that allowed for the detection of effects on actual cheating behavior rather than merely on self-reported cheating (by distinguishing between two measures of cheating: observed cheating behaviors via a confederate student and cheating on unsolvable questions). We preregistered our hypotheses, our research design, and the applied analytic strategy prior to data collection (https://aspredicted.org/7zv99.pdf).

\section{Method}

We conducted an experiment in which we manipulated both the appearance goals (independent variable 1) as well as the evaluation standards (result vs. process; independent variable 2). As dependent variables, we used two different cheating measures. All data and codes that support the findings of this study are provided in an open access repository (https://osf.io/548ry).

\section{Sample}

A total of 169 German university students ${ }^{1}$ (51 males, 116 females, 2 undisclosed; on average enrolled in their second year at university: $M$ [mean] $=1.98$ years, $S D$ [standard deviation $]=1.23$ years) participated in the experiment. We recruited participants by individually approaching university students on the campus. The participants were assured that their responses would remain confidential and would be used for scientific purposes only. The data were collected between May 13 and May 27, 2019.

\section{Procedure}

The participants were told that we had developed a "novel competence and personality test" that had to be tested in order to be used in an assessment center. The experiment was conducted in groups of five to eight students $(M=6.8$, $S D=0.9$ ). The groups were randomly assigned to one of four conditions based on the two independent variables (appearance goals and evaluation standards): (1) groups that received no appearance goal induction with a process-based evaluation standard $(n=39)$, (2) groups that received no appearance goal instruction with a result-based evaluation standard $(n=48)$, (3) groups that received an appearance goal instruction with a process-based evaluation standard $(n=41)$, and (4) groups that received an appearance goal instruction with a result-based evaluation standard $(n=41)$.

The experimenter induced appearance goals (independent variable 1) by requesting the participants to proceed as they would in an assessment center for a job that they would like to have and to try as hard as possible to make a good impression. The participants were also told that they would receive a $30 €$ voucher if they made a good impression on this test. The participants in the no-appearance goal condition were not instructed as described above. Instead, the examiner continued with the next part of the instruction straightaway. Herein, the experimenter manipulated the evaluation standards (independent variable 2) by informing the participants about the procedures and standards that would be applied to evaluate their performance. To put a focus on process-based evaluation standards, the experimenter told the participants that the strategies applied to solve the questions in the subsequent test would matter the most for the performance evaluation. The participants were told that they should focus on the correct approach to solve the task at hand. They were also told that the examiner would talk with them about their procedure after the test, and during the test they were asked which strategies they applied to solve the respective tasks. Conversely, the experimenter emphasized result-based evaluation standards by telling the 
participants that it primarily mattered whether their answers were correct. To yield comparability with the other condition, there were also interim questions throughout the knowledge test, which assessed participants' confidence in their answers being correct.

Immediately after the instruction, we asked the participants about their appearance goals, their extrinsic motivation, and the perceived evaluation standards. To hide our research focus, we asked these questions under the pretext of being interested in their current state of mind and included further questions (e.g., expectations regarding the test, current attentiveness). Among the participants, an in-group confederate student was present who pretended to participate in the study like the others but noted possible cheating behaviors of the other students instead. After the instruction, the examiner left the room under a pretext (receiving an urgent call) and told the participants that he would be back in 10 minutes. The participants were able to see the examiner coming back via the staircase before he could see the participants. As such, ample opportunities for cheating were given. At the end of the study, we asked the participants what they thought the study was about. Three participants mentioned that the study might have been about cheating and honesty. Following our preregistered procedure, we excluded these participants before running the final analyses. ${ }^{2}$

\section{Development of the Experimental Procedure in Two Preliminary Studies}

The research design was inspired by the experimental procedure developed by Daumiller and Janke (2019). In the original study, one participant and a confederate took part in what was believed to be a pretest of material for an assessment center. The participants completed a paper-pencil test and were provided with opportunities to cheat. The cheating behaviors were observed by the confederate. The procedure allowed for a suitable manipulation of appearance goals by emphasizing and incentivizing good impressions within the test. However, the original procedure was not very economical (only one participant per time slot) and was potentially prone to errors (paper- instead of computer-based assessment). That is why we further developed the procedure in two preliminary studies. In the first preliminary study, we created a computer-based version of the paper-and-pencil test and tested various new task types to measure cheating behavior. The results indicated that the study worked well as a computerized version and that the cover story was believable (none of the students detected our actual research aim). In the second preliminary study, we modified the procedure to allow for the assessment of data of multiple participants tested at the same time. We used the same manipulations as Daumiller and Janke (2019) and obtained very similar results, which we take as an indication of the validity of the multiple-participant paradigm. Detailed results from both preliminary studies can be found in the online supplement.

\section{Measurements}

Appearance Goals (Manipulation Check 1). We used the same two items developed by Daumiller, Dickhäuser, and Dresel (2019) to measure the participants' current appearance goals as were used in Daumiller and Janke (2019; "In the following test, my goal is to be perceived as competent" and "In the following test, my goal is that it is noticed how good I am;" Spearman-Brown=.78). The participants answered these items on a Likert-type scale ranging from 1 (do not agree at all) to 8 (agree completely).

Perceived Evaluation Standards (Manipulation Check 2). Using a slider, the participants were asked to mark between the two poles 0 (Using the correct strategies [Process]) and 100 (Reaching the right answer [Result]) what they were primarily concerned with regarding the following test.

Observed Cheating Behavior. The confederate student noted any unusual behaviors by the participants. In addition, we used screen-recording software to subsequently analyze what participants did on their computers. Based on both sources of information, each participant was classified by two raters as having cheated or not $(\kappa=1)$. This information was used as a dichotomous measure of cheating in the following analyses. Cheating behaviors included using the browser to search for the correct answers, peeking at other students' answers, asking other students for the correct answers, and using mobile phones to search for the correct answers.

Cheating on Unsolvable Questions. For some tasks (anagrams, visual thinking, figural thinking), we did not ask the participants for the exact answer but instead whether they were able to solve these tasks. Among these tasks, five questions were solvable and seven were unsolvable. For example, the figural thinking tasks entailed copying figures onto a separate paper without raising the pencil from the paper and without retracing any line. The participants were asked to state whether they had managed to do so (yes or no). In line with previous research employing such a measure (e.g., Lobel \& Levanon, 1988), we calculated the percentage of unsolvable tasks the participants indicated to have solved as a second cheating measure. The relative index allows for an intuitive understanding of our results and for directly comparing them with prior and future studies.

\section{Analyses}

We investigated the success of the experimental manipulations by conducting $t$-tests with IBM SPSS Version 25. For the main analysis, we first estimated two models for each of the cheating measures as dependent variables. We regressed each of them on the two independent variables and their 


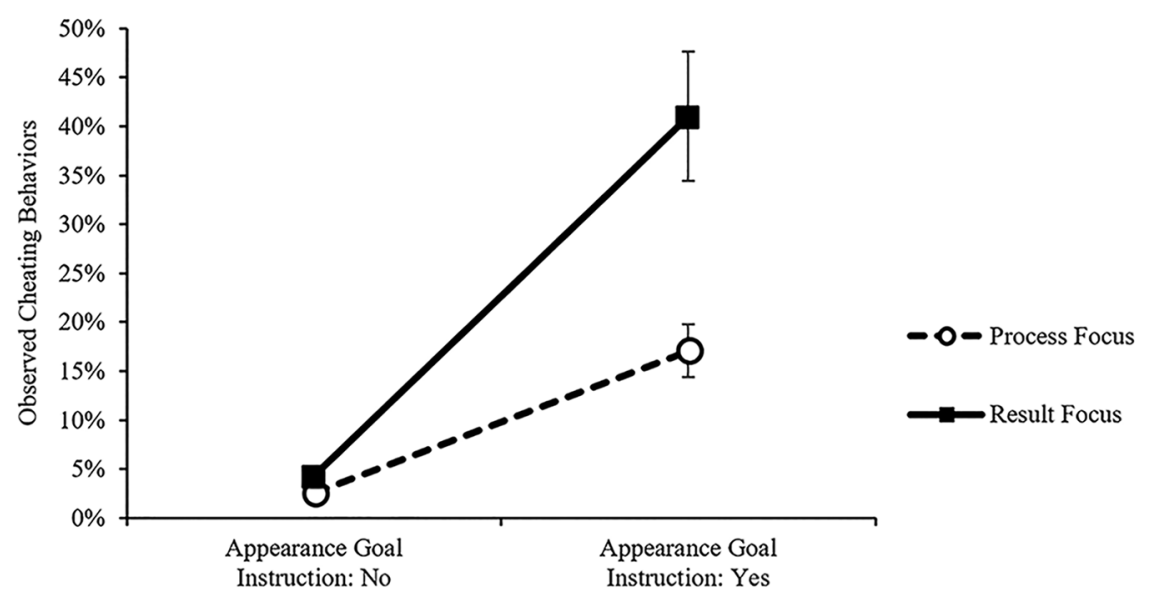

FIGURE 1. Effect of the experimental manipulation on observed cheating behaviors. Means and their standard errors for the percentage of participants who cheated under the different experimental conditions are presented.

interaction terms. We estimated both models with Mplus 8.1, which enabled us to handle the scale format and the distribution of the dependent variables appropriately. We used the WLSMV (weighted least square mean and variance adjusted) estimator for the analysis with the dichotomous dependent variable (observed cheating) and otherwise the MLR (robust maximum likelihood) estimator (for the analysis with the continuous cheating on unsolvable questions measure). Finally, we estimated one combined model (both cheating measures as dependent variables) that integrated both of the previous models. ${ }^{3}$

\section{Results}

In total, we observed that 27 of the 166 participants cheated $(16.3 \%)$. On average, $14 \%(S D=17 \%$; range: $0 \%$ to $86 \%$ ) of the unsolvable questions were marked as having been solved. A correlation matrix between all the variables reported in this study is provided as Supplemental Material. Comparing the appearance goals of the participants who did not receive the appearance goal instruction $(M=4.26$, $S D=1.57)$ with those of the participants who did $(M=5.43$, $S D=1.70)$ revealed a strong effect, confirming the success of the experimental manipulation $(T=-4.57, d f$ [degrees of freedom] $=164, p<.001$, Cohen's $d=.71) .{ }^{4}$ Likewise, comparing the perceived evaluation standards of the participants depending on whether the examiner focused on the process $(M=37.2, S D=23.5)$ or on the result $(M=73.3$, $S D=25.9)$ documented a strong effect, attesting that the manipulation of the second factor also worked well $(T=-9.39, d f=164, p<.001$, Cohen's $d=1.46)$.

For observed cheating (percentage of participants who cheated in no appearance goal + process group: $5 \%$, noappearance + product: $4 \%$, appearance + process: $17 \%$, appearance + product: $41 \%$ ) we found no significant main effect of the appearance goal condition (albeit this might be a result of impaired power, given the descriptive magnitude of the effect; $\beta=.29, p=.054$ ) or for the evaluation standard condition $(\beta=-.04, p=.81)$, while their interaction was statistically significant $(\beta=.30, p=.04)$. This indicates that students were only more likely to cheat when they were prompted to adopt appearance goals and the evaluation standard emphasized the product instead of the process (Figure 1).

For cheating on unsolvable questions (percentage of unsolvable questions that participants cheated on, in no appearance goals + process group: $M=14 \%, S D=15 \%$, no appearance goals + product: $M=11 \%, S D=14 \%$, appearance goals + process: $M=12 \%, S D=11 \%$, appearance goals + product: $M=21 \%, S D=25 \%$ ), we found similar results: no significant main effect for the appearance goal condition $(\beta=-.06, p=.48)$ or for the social norm condition $(\beta=-.08, p=.39)$, whereas their interaction was statistically significant $(\beta=.30, p=.006$; Figure 2$)$.

These findings of the single analyses were also evident in a combined moderation model (Figure 3), in which we observed the same moderation effects and saw that observed cheating behaviors and cheating on unsolvable questions were not substantially related.

\section{Discussion}

We investigated the role of students' appearance goals as well as the evaluation standards employed by teachers in students' engagement in cheating. Strengths of the study include the experimental design that we developed and confirmed with two preliminary studies, as well as the behaviorbased assessment of cheating (as opposed to self-reports). In line with our hypotheses, we found that neither appearance goals (value component) alone nor evaluation standards (expectancy component) alone led to increased cheating but that there was a significant interaction: Students cheated 


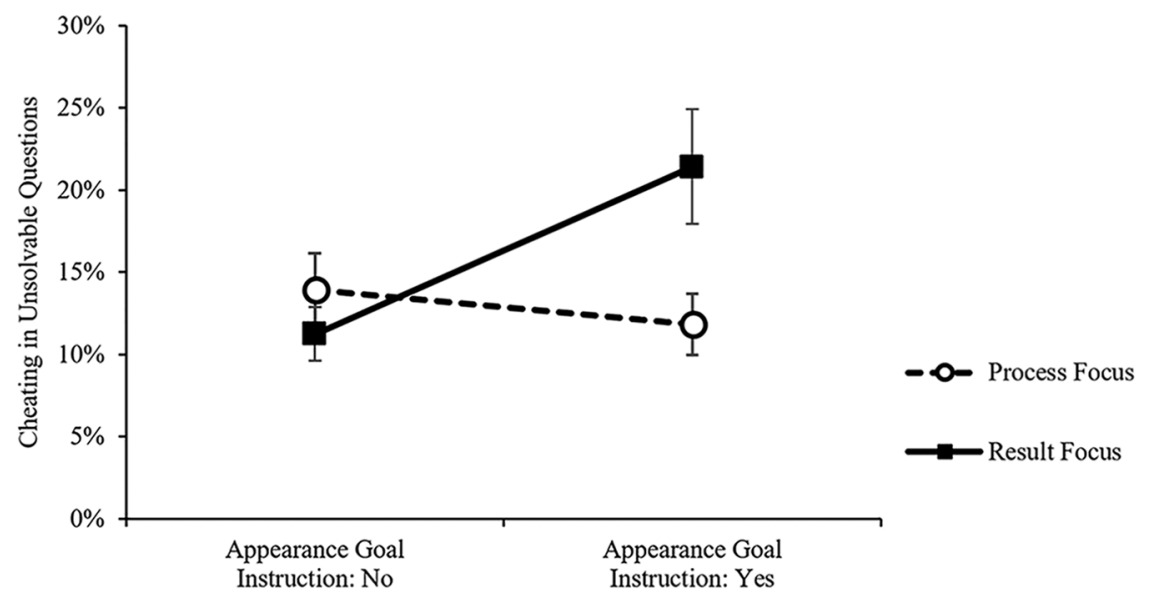

FIGURE 2. Effect of the experimental manipulation on cheating on unsolvable questions. Means and their standard errors for the percentage of unsolvable questions that participants cheated on in the different experimental conditions are presented.

twice as much when appearance goals were induced and they were told that their success would be evaluated strongly based on the results instead of the processes and strategies they used when solving the tasks. This multiplicative conjecture of appearance goals and results- versus processbased evaluation standards confirms the importance of focusing on further variables when investigating the effects of appearance goals on academic dishonesty. The observed findings yield implications for the theoretical understanding and application of achievement goal theory in the field of research on academic dishonesty.

\section{Implications for Motivational Theory}

Our results strengthen the claim that the diverse pattern of results regarding the association between appearance goals and academic cheating might be based on unobserved moderators (see Daumiller \& Janke, 2019; Janke et al., 2019). Identifying and understanding these moderators is necessary to gain a better understanding of the actual ramifications of appearance goals on academic dishonesty and the circumstances behind these effects. We conclude from these findings that theoretical frameworks beyond the scope of achievement goal theory are required to explain why appearance goals alone do not sufficiently predict engagement in cheating behavior. In the present work, we used the framework of expectancy-value theory (Wigfield \& Eccles, 2000) to identify evaluation standards as a potential moderator of the impact of appearance goals that is grounded in the achievement situation itself. We assumed that students' engagement in cheating behaviors would only increase when they endorsed appearance goals (associated with highly valuing success in the achievement situation at hand) and thought that there was a strong evaluation standard put on the results instead of the process (which is likely associated with a high expectancy of successfully conducting and getting away with cheating). The very similar findings regarding the two, rather distinct, aspects of cheating provide robust support for this theoretical assumption.

It is interesting to note that the baseline of cheating behavior was much lower than in a prior experimental study with similar methodology, in which cheating behavior was observed in $38 \%$ of the participants in the control condition (Daumiller \& Janke, 2019). This could be a function of multiple differences between the two studies, such as group composition (teacher trainees vs. students from unselected study programs, respectively), seating (in a closed room, next to another student vs. in a more open room, around a large table), or group size (two students vs. groups of five to eight students). Particularly, the larger group size may have reduced the likelihood of cheating, as it may have induced a stronger fear of detection or social punishment because of the increased number of observers who could negatively judge norm-deviant behavior. Although we do not think that these differences in the base rate of cheating influence the mechanisms investigated, future meta-analyses could profit by investigating such differences in cheating behavior as a function of study characteristics. These investigations could lead to the identification of additional environmental factors that could be manipulated for decreasing cheating behavior in achievement contexts. However, first, additional experimental studies on the impact of appearance goals on cheating are needed to provide the necessary foundation for meta-analyses.

\section{Practical Implications}

Beyond this, our findings give rise to meaningful practical implications for teaching personnel. Although it is, in principle, possible to change achievement goals (as also reflected in the experimental manipulations in the current study), this road constitutes a difficult avenue for many reallife achievement situations. Especially in high-stakes testing 


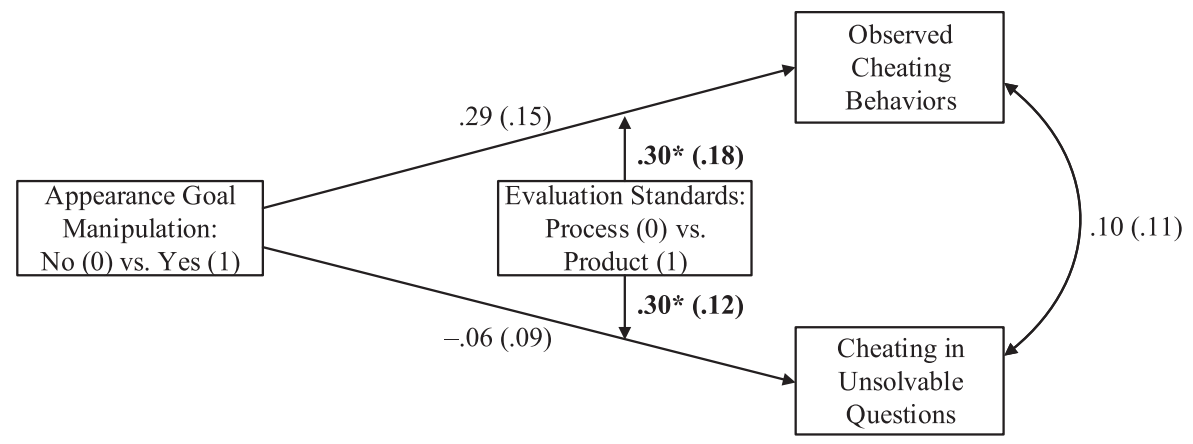

FIGURE 3. Combined moderation model. Standardized coefficients are presented, with standard errors in brackets. Statistically significant paths are boldfaced $(p<.05)$.

situations, where demonstrating competence is indeed of high importance, it may neither be advisable nor easily realizable to reduce appearance goals. At the same time, however, it is also of high interest to facilitate conditions that minimize the attractiveness of dishonest behaviors such as cheating. While this confronts the classical achievement goal theory approach with a paradox demand profile, our findings offer a glance into the means of modifying the respective achievement situations in ways that can prevent students with strong appearance goals from engaging in cheating behaviors.

When elaborating on potential ways to diminish cheating behavior, we have to acknowledge that the most successful way might be to eliminate cheating opportunities altogether through physical constraints (e.g., large space between students) and the undivided attention of the instructors. However, these means may not always be feasible or applicable as they require the right circumstances (e.g., a large room) and resources (e.g., a small ratio of students being tested to observing instructors). As such, it is important to provide means that are easily implementable and also helpful in reducing cheating behavior. With the results of our experimental study in mind, we believe that this could be achieved by informing students that the evaluation of their achievement will be largely based on the answer processes and strategies they applied rather than solely on the respective results. Instructors could rely on test items that allow process-based evaluations to enforce such an evaluation standard. To this end, open-ended questions are likely more suitable as they can better illustrate students' approaches to solving the questions while highlighting the importance of providing a consistent thought process - as opposed to multiple-choice items that likely indicate a focus on the result and only serve as an evaluation thereof.

Although it may not always be feasible to change all task types (especially in light of economic considerations) our findings document that the way tasks are framed and which evaluation standards are made salient can already have an effect. Drawing from the theoretical reasoning underlying the investigation at hand, it might also be worthwhile to focus on other aspects that influence the expectancy of indulging in and getting away with cheating in the achievement situation at hand (e.g., attentiveness of the instructor during the test). Identifying and following up on such moderators might be a worthwhile direction for future research. Furthermore, the experimental evidence should be supplemented with results from intervention studies that implement the proposed measures in real testing situations, as we still need to be cautious about whether our findings can be transferred to the realm of educational practice.

\section{Limitations and Implications for Future Research}

Despite its strengths, the current study has multiple limitations (especially regarding the postulated mechanisms) that need to be borne in mind when interpreting the results and that can serve to inform future research. First, our study is characterized by an experimental approach that allows differences in cheating behaviors to be attributed to the conducted manipulations. This is an important advancement over existing crosssectional studies that often only investigated self-reported cheating and make it difficult to ascribe the observed association between motivational factors and cheating to the motivational factors rather than to covariates of these variables. Although it is a strength of this approach that much of the variation that exists in everyday achievement situations can be controlled, the experiment only allows for the investigation of selected, isolated factors. As such, experiments have limitations when one is interested in more complex-yet possibly more realistic - interactions or in a more realistic estimate of effect sizes in the field. Despite this, experimental studies may still be considered as the most practical way to investigate actual cheating behavior. Here, field studies (e.g., observations, questionnaires) are difficult to implement (particularly to achieve a valid measure of cheating) and possibly even unethical as participants might run into problems when an observed academic misdemeanor is reported to academic authorities. Not reporting highly fraudulent behaviors after having observed them, however, could further foster a norm of social acceptance and also be unethical in itself. Finally, measuring cheating can become a problem if the participants know that 
the researchers are investigating cheating, which is largely deemed socially unacceptable. Thus, we believe that experimental studies may be the only way to investigate actual cheating, while field studies will mostly be limited to self-reported cheating because of practical and ethical considerations.

It should be noted that our study can only be considered as another step into a deeper understanding of the psychological mechanisms that explain under which circumstances appearance goals influence cheating behavior. Although we have proposed a theoretical model that we base our assumptions on and that serves well to explain our findings, some of the underlying mechanisms still require further investigation. Specifically, we explain the moderating effect of evaluation standards by assuming that a process-based evaluation of competence makes cheating more difficult and, thus, reduces the expectancy to succeed in cheating. Nevertheless, we have not investigated the participants' expectancy to succeed in cheating directly. This is a delicate issue, as explicitly asking about cheating prior to possible cheating behaviors could have informed the participants about our research question and impaired the validity of the design. However, asking about expectancies after the experiment would not have been a valid measure as the response would largely be influenced by the participants' experiences during the experiment. Following up on this by including further situated measures or different measurement approaches would therefore be another valuable direction for future research.

One intriguing finding of our study is that the magnitude of the association between performance goals and cheating differed on a descriptive level depending on the cheating behavior measured (stronger magnitude for observed cheating). Furthermore, both cheating measures were only weakly associated. This indicates that individuals likely differ in their approaches to cheating and that a person engaging in a certain form of cheating does not automatically imply that the individual will also engage in other kinds of cheating behavior. The factors that influence preferences for certain cheating behaviors are unclear at this point, which means that investigation into this issue is an interesting direction for future research.

Finally, in our experiment, we focused on appearance approach goals and did not consider avoidance motivation in greater detail. Regarding appearance avoidance goals (i.e., striving to avoid appearing incompetent), it is difficult to derive clear hypotheses. On one hand, appearance avoidance goals could be associated with the perspective that one is not able to perform well without cheating due to a lack of trust in one's capabilities, which in turn could facilitate cheating (Bong, 2008; Sicak \& Arslan, 2016). On the other hand, appearance avoidance goals are also linked to anxiety and could induce fear of detection, which could reduce cheating rates (Huang, 2011; Janke et al., 2016). In line with the latter explanation, empirical findings from field studies show that appearance avoidance goals are negatively associated with academic dishonesty when appearance approach goals are included in the model (Janke et al., 2019). Including the goal valence (i.e., their approach vs. avoidance focus) when investigating the circumstances under which appearance goals lead to cheating behaviors will be helpful in yielding a more comprehensive understanding of how individual appearance goals affect academic dishonesty.

\section{Conclusion}

The present study provides first insights into how the evaluation standards of performance tests can affect the impact of appearance goal striving on academic cheating. Our findings indicate that students cheat more when the situational context highlights the importance of appearance goals and the evaluation standards are strongly based on the results and not the processes that students use during task engagement. The mechanisms behind the observed interaction warrant further investigation, and our findings should be transferred to more naturalistic settings (particularly as we experimentally induced the achievement goals instead of studying their naturally occurring variability, which might evoke different effects). Nevertheless, the results provided imply that practitioners who develop tests for high-stakes testing should use evaluation practices (and tasks) that also reward the strategies used by students to achieve their answer instead of solely the correct results.

\section{Authors' Note}

Both authors have equally contributed to the article and have split first authorship.

\section{Acknowledgments}

We thank Anne Felbach, Selena Seiler, and Daniele Crivaro for their help during data collection.

\section{Notes}

1. We planned to recruit 171 students. However, having reached 169 students, it was not feasible to conduct the experiment again with 2 more participants as the whole experiment would have been needed to be set up again on another day and there would have been an unequal group size assignment.

2. One of the three participants was from the no appearance goal + product focus group, and the other two were from the appearance goal + product focus group. For one of the latter, we observed cheating behaviors during the test, but for the others, we did not. Additional analyses including the three participants yielded no substantial differences in the result pattern.

3. Besides these preregistered analyses, we conducted a series of additional analyses to confirm the robustness of our findings. First, we conducted a set of simple 2F-ANOVAs in SPSS. Second, as the students were organized in groups, statistical dependence of the cheating data was possible. We investigated this by calculating intraclass correlation coefficients (ICCs) and found a small effect of group membership on observed cheating behavior (ICC1 $=.09$ ) but not on cheating on unsolvable questions (ICC1 $<.001$ ). To determine whether this had an impact on our findings, we reran 
our analyses accounting for between-group variance by using the "type = complex" command in Mplus. Finally, given that we observed higher cheating rates for males than for females, we also controlled for participants' gender. These additional sets of analyses yielded very similar results to those presented in the text.

4. Furthermore, we wanted to test whether this experimental manipulation focused on the goals and not on extrinsic motivation. We measured the participants' extrinsic motivation with a single, self-constructed item ("In the following test, I mainly care about the money") that was to be answered on the same scale as the appearance goals. Comparing participants again, this time regarding extrinsic motivation, documented no statistically significant differences between the groups in extrinsic motivation $(T=0.99, d f=164, p=.32)$. We also explored the role of self-efficacy beliefs for goal pursuit as we seek to follow up on this by investigating the moderating role of self-efficacy beliefs in the effect of performance goals on cheating in future work (see preregistration). An analysis of variance with appearance goal induction (yes or $n o$ ) as a factor and strength of participants' self-efficacy beliefs as a covariate indicated statistically significant main effects as well as a significant interaction $\left(F=8.00, d f=1, p=.005, \eta^{2}=.05\right)$, supporting the finding that individuals with stronger self-efficacy beliefs adopt stronger appearance approach goals than individuals with weaker selfefficacy beliefs.

\section{References}

Anderman, E. M. (2007). The effects of personal, classroom, and school goal structures on academic cheating. In E. M. Anderman, \& T. B. Murdock (Eds.), Psychology of academic cheating (pp. 87-106). New York, NY: Academic Press.

Anderman, E. M., \& Koenka, A. C. (2017). The relation between academic motivation and cheating. Theory Into Practice, 56, 95-102. doi:10.1080/00405841.2017.1308172

Bong, M. (2008). Effects of parent-child relationships and classroom goal structures on motivation, help-seeking avoidance, and cheating. Journal of Experimental Education, 76, 191-217. doi:10.3200/JEXE.76.2.191-217

Brookhart, S. M., Guskey, T. R., Bowers, A. J., McMillan, J. H., Smith, J. K., Smith, L. F., . . Welsh, M. E. (2016). A century of grading research: Meaning and value in the most common educational measure. Review of Educational Research, 86, 803-848. doi:10.3102/0034654316672069

Darnon, C., Butera, F., \& Harackiewicz, J. M. (2007). Achievement goals in social interactions: Learning with mastery vs. performance goals. Motivation and Emotion, 31, 61-70. doi:10.1007/ s11031-006-9049-2

Daumiller, M., Dickhäuser, O., \& Dresel, M. (2019). University instructors' achievement goals for teaching. Journal of Educational Psychology, 111, 131-148. doi:10.1037/edu0000271

Daumiller, M., \& Janke, S. (2019). Effects of performance goals and social norms on academic dishonesty in a test [Online]. British Journal of Educational Psychology. doi:10.1111/bjep.12310

Dickhäuser, C., Buch, S. R., \& Dickhäuser, O. (2011). Achievement after failure: The role of achievement goals and negative selfrelated thoughts. Learning and Instruction, 21, 152-162. doi:10.1016/j.learninstruc.2010.01.002

Dietrich, J., Viljaranta, J., Moeller, J., \& Kracke, B. (2017). Situational expectancies and task values: Associations with students' effort. Learning and Instruction, 47, 53-64. doi: 10.1016/j.learninstruc.2016.10.009
Elliot, A. J. (2005). A conceptual history of the achievement goal construct. In A. J. Elliot \& C. S. Dweck (Eds.), Handbook of competence and motivation (pp. 52-72). New York, NY: Guilford Press.

Finn, K. V., \& Frone, M. R. (2004). Academic performance and cheating: Moderating role of school identification and selfefficacy. Journal of Educational Research, 97, 115-121. doi:10.3200/JOER.97.3.115-121

Grant, H., \& Dweck, C. S. (2003). Clarifying achievement goals and their impact. Journal of Personality and Social Psychology, 85, 541-553. doi:10.1037/0022-3514.85.3.541

Huang, C. (2011). Achievement goals and achievement emotions: A meta-analysis. Educational Psychology Review, 23, 359-388. doi:10.1007/s10648-011-9155-x

Janke, S., Daumiller, M., \& Rudert, S. C. (2019). Dark pathways to achievement in science: Researchers' achievement goals predict engagement in questionable research practices. Social Psychological and Personality Science, 10, 783-791. doi:10.1177/1948550618790227

Janke, S., \& Dickhäuser, O. (2019). A neglected tenet of achievement goal theory: Associations between life aspirations and achievement goal orientations. Personality and Individual Differences, 142, 90-99. doi:10.1016/j.paid.2019.01.038

Janke, S., Nitsche, S., Praetorius, A. K., Benning, K., Fasching, M., Dresel, M., \& Dickhäuser, O. (2016). Deconstructing performance goal orientations: The merit of a dimensional approach. Learning and Individual Differences, 50, 133-146. doi:10.1016/j.lindif.2016.08.013

Jordan, A. E. (2001). College student cheating: The role of motivation, perceived norms, attitudes, and knowledge of institutional policy. Ethics \& Behavior, 11, 233-247. doi:10.1207/ S15327019EB1103_3

Krou, M. R., Hoff, M., Hewett, E., \& Fong, C. J. (2019, April). To cheat or not to cheat: What is the motivation? A meta-analytic investigation. Paper presented at the American Education Research Association, Toronto, Ontario, Canada.

Lobel, T. E., \& Levanon, I. (1988). Self-esteem, need for approval, and cheating behavior in children. Journal of Educational Psychology, 80, 122-123. doi:10.1037/0022-0663.80.1.122

Lüftenegger, M., Van De Schoot, R., Schober, B., Finsterwald, M., \& Spiel, C. (2014). Promotion of students' mastery goal orientations: Does TARGET work? Educational Psychology, 34, 451-469. doi:10.1080/01443410.2013.814189

Marsden, H., Carroll, M., \& Neill, J. T. (2005). Who cheats at university? A self-report study of dishonest academic behaviours in a sample of Australian university students. Australian Journal of Psychology, 57, 1-10. doi:10.1080/00049530412331283426

McCabe, D. L. (2005). It takes a village: Academic dishonesty and educational opportunity. Liberal Education, 91(1), 26-31.

Megehee, C. M., \& Spake, D. F. (2008). The impact of perceived peer behavior, probable detection and punishment severity on student cheating behavior. Marketing Education Review, 18, 5-19. doi:10.1080/10528008.2008.11489033

Murayama, K., Elliot, A. J., \& Friedman, R. (2012). Achievement goals. In R. M. Ryan (Ed.), The Oxford handbook of human motivation (Oxford Library of Psychology, pp. 191-207). New York, NY: Oxford University.

Nagengast, B., Marsh, H. W., Scalas, L. F., Xu, M. K., Hau, K. T., \& Trautwein, U. (2011). Who took the "x" out of expectancy-value 
theory? A psychological mystery, a substantive-methodological synergy, and a cross-national generalization. Psychological Science, 22, 1058-1066. doi:10.1177/0956797611415540

Ramberg, J., \& Modin, B. (2019). School effectiveness and student cheating: Do students' grades and moral standards matter for this relationship? [Online]. Social Psychology of Education. doi:10.1007/s11218-019-09486-6

Rettinger, D. A., Jordan, A. E., \& Peschiera, F. (2004). Evaluating the motivation of other students to cheat: A vignette experiment. Research in Higher Education, 45, 873-890. doi:10.1007/ s11162-004-5952-0

Rudert, S. C., Ruf, S., \& Greifeneder, R. (2019). Who to punish? How observers sanction norm-violating behavior in ostracism situations [Online]. European Journal of Social Psychology. doi:10.1002/ejsp.2606

Sicak, A., \& Arslan, A. (2016). The relation between prospective teachers' goal orientations and academic e-dishonesty. Universal Journal of Educational Research, 4, 1660-1666. doi:10.13189/ujer.2016.040718

Teixeira, A. A., \& Rocha, M. F. (2010). Cheating by economics and business undergraduate students: An exploratory international assessment. Higher Education, 59, 663-701. doi:10.1007/s10734009-9274-1

Trautwein, U., Marsh, H. W., Nagengast, B., Lüdtke, O., Nagy, G., \& Jonkmann, K. (2012). Probing for the multiplicative term in modern expectancy-value theory: A latent interaction modeling study. Journal of Educational Psychology, 104, 763-777. doi:10.1037/a0027470

Wigfield, A., \& Eccles, J. S. (2000). Expectancy-value theory of achievement motivation. Contemporary Educational Psychology, 25, 68-81. doi:10.1006/ceps.1999.1015

\section{Authors}

MARTIN DAUMILLER is an assistant professor in the Department of Psychology at the University of Augsburg, Germany. His research interests include goals and achievement goal theory, academic dishonesty, cognition and behavior of university scholars, and learning with digital media in the tertiary sector.

STEFAN JANKE is a postdoc in the Department of Pedagogical Psychology at the University of Mannheim, Germany. His research interests include higher education, faculty motivation, and social psychology of education. 